\title{
IMPACT OF OBESITY ON RISK OF CANCER
}

\author{
Karolina Krupa-Kotara, Dominika Dakowska \\ Department of Epidemiology, School of Health Sciences, Medical University of Silesia in Katowice, Katowice, Poland
}

\section{SUMMARY}

Epidemiological data consistently show sustained upward trend in the incidence of cancer. The International Agency for Research on Cancer (IARC) estimated that in 2018 more than 18 million people were diagnosed with cancer, and in about 9.6 million cases cancer could be the cause of death. At the same time, an increasing percentage of overweight and obesity is observed in both adults and children. According to the World Health Organization (WHO), in 2016 over 1.9 billion (39\% of the population) of adults were overweight, of which over 650 million (13\%) were obese. Obesity is closely related to the risk of cancer development. Excessive body weight is considered as a second risk factor for the development of specific cancers after smoking. According to IARC data, high BMI index may be the cause of up to half a million cancer cases per year. In addition, overweight and obesity contribute to increased mortality due to malignancies - it is estimated that they are the cause of death in $20 \%$ of women and in $14 \%$ of men. Data from the American Cancer Society indicate increased mortality due to pancreatic cancer, liver cancer, multiple myeloma, and Hodgkin's lymphoma in connection with the occurrence of excessive body weight.

The study tried to confirm the influence of obesity on the risk of cancer. To achieve this, risk factors such as excessive adipose tissue, chronic inflammation, hyperinsulinaemia and hyperglycaemia, sex steroids, and microbiome were taken into account. In addition, the study presents a simple method of estimating the risk quotient of chances of developing malignant tumors in people with excessive body mass and methods of cancer disease prevention.

According to the current state of knowledge, the modification of factors significantly affecting the risk of falling ill may contribute to reducing the risk of cancer. In view of the above, the importance of the problem of overweight and obesity in society and their impact on the occurrence of some types of cancer should be emphasized. Thus, the understanding of excessive body weight as a serious health and social problem sets one of the priorities in the area of public health.

Key words: obesity, cancer, excessive body weight, risk factors

Address for correspondence: K. Krupa-Kotara, Department of Epidemiology, School of Health Sciences, Medical University of Silesia in Katowice, Piekarska 18, 41-902 Bytom, Poland. E-mail: kkrupa@sum.edu.pl

https://doi.org/10.21101/cejph.a5913

\section{INTRODUCTION}

The epidemiological data shows a constant trend in increasing amount of people getting sick with cancer. The International Agency for Research on Cancer (IARC), part of the World Health Organization (WHO), estimated in their Globocan 2018 report, that over 18 million people were diagnosed with cancer, and in about 9.6 million cases cancer may have been a cause of death. In the world, there are over 40 million people, who were diagnosed with cancer during last 5 years. IARC predicts that during their lifespan 1 in 6 women and 1 in 5 men will become oncological patients. Constant rise in people getting sick with cancer is attributed to aging of societies throughout the world. Report states that $23.4 \%$ of Europeans will be sick with cancer and $20.3 \%$ of deaths caused by cancer will occur. According to the WHO, cancer is the second most common cause of death in the world. However, already in 28 European countries of the Western region, cancers have replaced cardiovascular diseases as the leading cause of premature deaths. The most commonly diagnosed types of cancer in 2018 were: lung cancer, colorectal cancer and breast cancer, of which the latter two types are obesity induced (1). A constant rise in overweight and obesity among both adults and children is simultaneously observed. The WHO states that in 2016 over 1.9 billion (39\% of the population) adults were overweight, and over 650 million (13\%) of them were obese (2). The number of obese children in the age between 5-19 years rose tenfold during last four decades. At present, there are 430 million children with excessive body weight (3).

\section{Risk Factors of Obesity}

According to WHO definition, obesity is an abnormal or excessive fat accumulation that presents a risk to health. The development of obesity is influenced by genetic factors that play an important role, however, they are only predispositions that can be revealed only after the environmental factors have been triggered - bad eating habits, reduced physical activity, stress, as well as other causes such as hormonal imbalance or the use of certain drugs. Simple obesity, which is about $90 \%$ of cases, is caused by excessive supply of food in relation to energy expenditure. Secondary obesity may occur in the course of endocrinopathy, genetically determined syndromes, damage to the hypothalamus by inflammation, injuries of the central nervous system, or as a result of taking certain medications (3). 
Impact of Excessive Body Weight on Risk of Cancer

Risk of cancer is strictly tied to obesity. Excessive body weight is considered second major factor of getting sick with cancer, after smoking tobacco. According to IARC data, high BMI value may be a cause of almost half million cases of cancer per year. Study conducted by IARC emphasizes that excessive bogy weight caused about 3.6\% of all diagnosed cancer cases in 2012 (1).

According to IARC, obesity in Europe is the cause of $11 \%$ of cases of colon cancer, $9 \%$ of postmenopausal breast cancer, $39 \%$ of endometrial cancer, $25 \%$ of kidney cancer, and $37 \%$ of oesophageal cancer. In addition, according to the American Cancer Society (ACS), overweight and obesity are associated with mortality from liver cancer, pancreatic cancer, Hodgkin's lymphoma, and multiple myeloma (4). In the IARC report, the evidence was supported by data on the cause and effect relationship between overweight and obesity and the occurrence of these cancers (5).

It is estimated that overweight and obesity are responsible for around $20 \%$ of all cancers and are the cause of death from malignancies in $14 \%$ of men and $20 \%$ of women (6).

Obesity, besides raising risk of cancer, is also an adverse factor to patient's prognosis. It makes establishing correct doses for chemotherapy harder and can have a negative effect on used anticancer treatment (high probability of metastasis, lessening the effects of treatment or heightened danger of unwanted effects). Above that, overweight and obesity contribute to increase in mortality caused by malignant tumour - it is estimated that they make up to deaths of $20 \%$ women and $14 \%$ of men. ACS data shows increased mortality rate caused by pancreatic cancer, hepatocellular carcinoma, multiple myeloma, and Hodgkin's lymphoma linked to excessive body weight (7).

\section{Mechanisms Linking Obesity to Induction of Neo- plastic Processes}

Pathomechanisms responsible for cancerous processes are very complex in case of obese people. First of all, they include higher concentration and bioavailability of insulin and insulin-like growth factor IGF-1, incorrect secretion of adipokines, chronic inflammation, higher concentration of locally produced sex steroids (e.g. estrogen), changed immune response, oxidative stress, and disorders in intestinal microflora's composition (8).

\section{Fat Tissue and Cancer}

Fat tissue is not only an energy storage, but it also takes a role of an endocrine organ responsible for secretion of bioactive substances. This tissue is diverse not only morphologically, but also functionally, and adipokine secretion depends on location. In addition to adipocytes, fat tissue's composition consists of macrophages, lymphocytes, preadipocytes, fibroblasts, endothelial cells, and their amount depends on the type of tissue and degree of obesity. Fat tissue is spread around the whole body, and we can separate few of its main locations: gluteal-femoral, ventral visceral and ventral subcutaneous, in which accumulates about $70 \%$ of fat. On this basis we can separate two types of obesity: gynoid - or pear-shaped obesity, and android - or apple-shaped obesity. The ventral visceral fat tissue is especially dangerous to one's health - it plays a key role in development of insulin resistance, type 2 diabetes, and illnesses of cardiovascular system. Its negative action is associated with releasing free fatty acids and adipokines into the portal circulation. Ventral visceral fat tissue is more metabolically and hormonally active compared to ventral subcutaneous fat tissue (9).

In human organism fat tissue is divided into two types: white adipose tissue (WAT) and brown adipose tissue (BAT). The newest research show that there is also pink adipose tissue, which forms in pregnant women and during lactation. Brown adipose tissue utilizes lipids and produces energy, it is responsible for heat production and thermogenesis. Its malfunctioning may favour obesity. Constant stimulation of obese people's sympathetic system causes handicap of thermogenesis and excessive fat storage. White adipose tissue storages triglycerides, that means energy, which is used during increased energy expenditure (9). Triglycerides undergo lipolysis and are released into the bloodstream in form of free acids, and then they can be used for energy production. During metabolic diseases white adipose tissue does not react correctly to changed energetic request, which leads to increased production of adipokines and cytokines (10). Excessively accumulating WAT promotes proliferation of cancerous cells, granting them access to free fat acids, as cancerous cells adjust to changes in energy request, as to have constant access to fuel needed for proliferation. In obese people size and number of adipocytes increase due to excessive energy storage. Adipocyte hypertrophy is linked to insulin resistance and release of proinflammatory cytokines. White adipose tissue is of great endocrine importance. Adipokines produced by it show pleiotropic activity and take part in, among others, immune responses, lipides and glucose metabolism, angiogenesis, and coagulation processes (11).

Leptin is one of main, most important adipokines - it regulates food intake and, when it binds with it is ObR receptor, it influences hypothalamus neurons, thus influencing body's energy management. It shows double action - its high concentration works anorectic, while its low concentration stimulates appetite. Concentration of this adipokines strongly correlates with presence of adipose tissue. Increase in adipose tissue, especially ventral visceral, along with highly caloric diet is tied to increase in leptin concentration, while reduction of body weight leads to decrease in its concentration. Due to mechanisms that aid migration and proliferation of cells, like modulation of the signal path JAK/ STAT, stimulation of inflammation, increase of hypoxia induced factor and angiogenesis factors, leptin favours cancerous processes (12). It may modulate tumour's growth through increasing vascular endothelial growth factor (VEGF) expression, key stimulant of angiogenesis. It acts mitogenously on epithelial cells of the gastrointestinal tract and intensifies proliferation of ovarian, oesophageal, pancreatic, stomach, and lung cancer cells. In-vitro studies show that when leptin activates specific ObR receptors, it stimulates proliferation and survival of large intestine, breast and endometrium's cancerous cells. The anti-inflammatory adipokine acting opposite to leptin, produced by mature fat cells, is adiponectin, whose in-blood concentration is lower in people with visceral obesity due to oxidative stress, hypoxia and the release of pro-inflammatory cytokines IL- 6 and TNF- $\alpha$ inhibiting its production $(11,13)$. Adiponectin reduces the effect of the insulin/IGF-1 axis, which is the basic molecular pathway in the progression and initiation of tumours. This thesis is confirmed by higher cancer mortality in obese patients with type 2 diabetes, which may be a result of high concentrations of IGF-1 and 
insulin (10). It also affects the decreased proliferation of tumour cells and inhibits tumour growth, and by lowering the expression of VEGF blocks angiogenesis. It has been shown that decreased adiponectin concentrations observed in obesity can be one of the mechanisms linking obesity with the development and progression of cancer. Studies indicate a negative correlation between adiponectin concentration and the risk of endometrial, breast, pancreatic, prostate, colorectal, and liver cancer (12). Visfatin also affects carcinoma to some extent, however, its role is still not fully understood. On mouse cells it has been shown to induce oxidative stress and the synthesis of free oxygen radicals. In addition, it indirectly affects the synthesis of many proinflammatory cytokines, increasing inflammation and favours the development of angiogenesis. There are also reports on the role of visfatin in the development of gastrointestinal tumours (its increased concentration is observed in the rectal tumour) and prostate cancer. Its increased expression is shown by cells that are resistant to chemotherapy. There is evidence of resisting participation in the activation of the inflammatory process. It stimulates the production of TNF- $\alpha$, IL-12 and IL- 6 and weakens the anti-inflammatory effect of adiponectin. The concentration of resisting increases in patients who undergo a chronic inflammatory process inducing cancer in ovarian cancer (14).

\section{Chronic Inflammation}

Excessively accumulating visceral fat causes chronic inflammation, which leads to improper activation of proinflammatory signals and cytokine production. This condition is compounded by the release of free fatty acids into circulation and excitation of the immune system, especially macrophages. Macrophages in adipose tissue in obesity are enlarging, transforming into pro-inflammatory macrophages, that secrete cytokines, e.g. alpha tumour necrosis factor (15). The inflow of these cells to adipose tissue is proportional to obesity, and reduction of body mass reduces their number in the tissue. Free fatty acids activate $\mathrm{NF}-\kappa \mathrm{B}$ transcription factor, which plays an important role in epithelial carcinogenesis, is associated with insulin resistance, and indirectly stimulates cancer cell proliferation and apoptosis and inflammation. Its activation is associated with angiogenesis and metastasis. NF- $\kappa \mathrm{B}$ transcription alone is activated in response to growth factors and proinflammatory cytokines. Excessive levels of NF-kB activity may affect the development of gastric, lung, colorectal cancer, breast cancer, or squamous cell carcinoma of the head and neck $(15,16)$.

Fat tissue also secretes proinflammatory interleukins 1 and 6 , tumour necrosis factor alpha, monocyte chemoattractant 1 (MCP-1), and C-reactive protein (CRP), which may cause local inflammation in adipose tissue and act systemically on other organs (13). There are numerous examples of local inflammation associated with cancer risk, e.g. the association of non-alcoholic steatohepatitis with liver cancer (17). Chronic gastro-oesophageal reflux leading to the development of Barrett's oesophagus and oesophageal adenocarcinoma (8), a relationship between chronic cholelithiasis and cholecystitis with gallbladder cancer (18), or stomach cancer caused by inflammation of the mucous membrane. $\mathrm{C}$-reactive protein is the main diagnostic exponent of inflammation. It has been proven that high levels of CRP increase the risk of developing ovarian cancer in women with excessive body mass (14). A proinflammatory cytokine secreted by adipocytes whose elevated levels are found in obese subjects is TNF- $\alpha$. Its pro-inflammatory activity plays a role in all stages of oncogenesis, which include transformation and proliferation of tumour cells, survival, invasion, angiogenesis, and metastases. A positive correlation was found between alpha tumour necrosis factor and the development of liver cancer and colorectal cancer (17).

Another cytokine produced by adipose tissue belonging to the group of proinflammatory cytokines is IL-6, whose increased concentrations are observed in obese people. It is a key modulator in carcinogenesis associated with inflammation and is often produced by cancer cells. It regulates the expression of genes involved in various stages of tumour growth and progression through the JAK/STAT signalling pathway. It has been shown that elevated IL- 6 concentration correlates with tumour aggressiveness and poor prognosis in patients with ovarian, breast, kidney, lung, and colorectal cancer $(14,17)$. Interleukin-1 can lead to activation of transcription factors, such as NF-kB and AP1 , and promote genes that regulate the mechanisms of survival, proliferation and tumour angiogenesis. In inflammation associated with obesity, IL-1 indirectly influences the increase in the expression of angiogenic VEGF necessary for tumour growth and metastasis (17). Another pro-inflammatory mediator important in the treatment of cancer as well as in the prevention of cancer is cyclooxygenase-2 (COX-2). Its overexpression is visible in most cancers of the pancreas, breast, large intestine, bladder, stomach, skin, head, and neck (16).

Chronic inflammation observed in obesity predisposes to certain types of cancer by creating a tissue environment that produces oxidative stress, stimulates DNA damage, increases cell proliferation, and inhibits apoptosis (8). In inflammatory cells migrating to the site of inflammation, reactive oxygen and nitrogen forms are generated that induce DNA mutations in normal epithelia (15). The development of inflammation in obesity is also influenced by the hypoxia of adipocytes, which stimulates the secretion of leptin and interleukin-6 (12).

Many studies indicate chronic inflammation as the main risk factor for cancer development and progression. It is estimated that infections and chronic inflammatory reactions are the cause of $15-25 \%$ of deaths from cancer in the world (estimated 15-25\% of cancer-related deaths worldwide can be attributed to infections and chronic inflammatory reactions) (19).

\section{Hyperinsulinaemia and Hyperglycaemia}

Excessive body weight and chronic inflammation, especially visceral obesity, is the strongest factor determining insulin resistance, the most common effect of which is hyperinsulinaemia.

The role of insulin and insulin-like growth factor IGF-1 is the basis for understanding why these diseases, which together accompany primarily abdominal obesity, increase the risk of innumerable types of cancer. Insulin is a mitogenic hormone. Its high concentration in the blood by activating MAP-kinase stimulates cell proliferation, which can cause bladder or colon cancer in women and non-Hodgkin's lymphoma or liver cancer in men. Insulin has ability to bind to mitogenic hybrid receptors as well as to the IGF-1 insulin-like growth factor receptors. It can inhibit apoptosis and stimulate IGF-1 synthesis, thereby promoting tumorigenesis (20). In obese people with hyperinsulinaemia, 
IGF-1 levels are elevated and there is a reduced synthesis of IGFBPI and IGFBP2-binding proteins related to insulin-like growth factors. This affects cell surface receptors and influences tumour development. Insulin-like growth factor is involved in the regulation of the ras gene. Mutations of this gene are found in the early stages of cancer development. This mechanism plays a special role in the development of colorectal cancer and breast cancer, in which nearly $90 \%$ of cells have been shown to have increased expression of IGF. Insulin and IGF levels in breast cancer cells are tenfold higher compared to healthy cells $(9,10)$.

Hyperinsulinaemia is conducive to the rapid development of cancer of the large intestine, pancreas, liver, endometrial cancer, and breast cancer in postmenopausal women (20). It can induce a neoplastic process through increased metabolic cell activity that damages DNA and leads to mutagenesis. High insulin concentrations in in vivo studies on kidney and colon cells have resulted in increased mitochondrial production of reactive oxygen species (ROS), resulting in DNA rupture. Such damaged genetic material is mutagenic and can cause tumour growth (21).

The characteristic of cancer cells is the ability to constantly divide, and their metabolism is aimed at constant mass increase, which is possible with access to glucose - the basic source of energy. Therefore, these cells require greater uptake of glucose, which they consume in the process of aerobic glycolysis. Then, from 1 mole of glucose, it is possible to produce 36 moles of ATP. This fact is important in explaining the relationship between hyperglycaemia in people with diabetes and the higher incidence of cancer in this group (7). In many studies, the relationship between fasting glucose and carcinogenesis has been documented. The risk of cancer as well as the risk of death from cancer in both men and women increases with an increase in fasting blood glucose levels by up to $1 \mathrm{mmol} / \mathrm{l}(15)$.

\section{Sex Steroids}

The BMI of both women and men strongly correlates with the concentrations of sex steroids. Visceral fat as an endocrine organ, affects the synthesis and bioavailability of sex hormones, which are also responsible for the process of cancer. It produces estrogen - estrone and estradiol, which stimulate insulin-like growth factor IGF-1 receptors and support cell proliferation. High levels of estrogen or progesterone deficiency are important in the development of breast cancer in postmenopausal women and endometrial cancer - they increase the production of free oxygen radicals and damage genetic material. The study also assesses the relationship between sex steroids and pre-menopausal cancer, but the data is inconsistent and further research is needed. However, it has been proven that among women struggling with polycystic ovarian syndrome, excess androgens and progesterone deficiency increase the risk of cancer before the menopause (8). In the group of obese women there is a higher risk of developing postmenopausal breast cancer due to high levels of sex hormones - testosterone, dehydroepiandrosterone (DHEA) and its sulphate (DHEAS), estrone and total estradiol, and a smaller synthesis of sex hormone binding globulin (SHBG), i.e., proteins that binds sex steroids. Low concentration of this globulin with simultaneous high concentration of sex hormones may be a consequence of the development of endometrial and breast cancer. In adipose tissue, the expression of aromatase enzymes is increased, which convert androgens into estrogens and transform the less active forms of these hormones (androstenedione and estrone) into more active testosterone and estradiol. In postmenopausal women the rate of conversion of androgens to estrogens is significantly higher among women with obesity (16).

In obese men there is a significant reduction in testosterone concentration and higher estrogen concentration. Abnormalities in the concentration of sex steroids may contribute to the development of colorectal cancer and prostate cancer, however, the aetiology connecting sex hormones with advanced prostate cancer is not fully understood. In men with a high BMI index there is a reduced level of androgens, which play an important role in the normal growth and differentiation of prostatic epithelial cells. Hence the hypothesis that the lower bioavailability of testosterone may promote neoplasia in prostate cancer (8).

\section{Microbiota in Obesity and Cancer}

Many studies indicate interactions between intestinal microflora and body composition and metabolites of nutrients. Diet and body composition affect the functioning and composition of the microflora, and this in turn affects the physiology and state of the host organism. Searching for the causes of obesity so common in the population, more often attention is paid to the dysbiosis of the intestinal microflora, depending on the BMI. Recent reports emphasize the important role of the microbiome in the aetiology of obesity and the development of gastrointestinal tumours. Intestinal microflora can initiate inflammatory, hormonal, and immunological processes responsible for neoplastic formation and abnormal multiplication of intestinal epithelial cells. It can be a factor determining the formation of metastases and affect the response to treatment. Recent studies have shown that some bacteria can improve the effects of anti-cancer drugs and immunotherapeutic drugs (22).

The human digestive tract is inhabited by approximately $10^{14}$ bacteria (800 species and 7000 strains), however, their number and composition of flora is different for every human being. There are five main types of bacteria in the gut, of which $90 \%$ are Bacteroidetes and Firmicutes. Their number depends mainly on diet and body composition. BMI above $30 \mathrm{~kg} / \mathrm{m}^{2}$ strongly correlates with higher number of Firmicutes bacteria and decrease in the number of Bacteroidetes bacteria (23).

It should be emphasized that in the reduction of body mass the composition of the intestinal microflora also changes - the number of Mollicutes (Firmicutes) decreases, and a similar fact was also observed after the bariatric treatment. Bacteria of the intestinal microflora through the mechanism of bile acids, primarily deoxycholic acid, can affect the development of colorectal cancer. One mechanism is the suppression of p53 protein, which limits its ability to repair DNA. Intestinal microflora may also promote the metabolism of estrogen, thereby increasing estradiol concentration and accelerating the formation of endometrial cancer or breast cancer after menopause. In obesity, there is a decrease in the diversity of bacteria in the microflora, which increases the concentration of CRP in the blood and increases the number of leukocytes. Disorders in the intestinal microflora accompanying obesity may induce chronic inflammation of the adipose tissue, which determines the development of obesity-induced cancers. Numerous studies also suggest a link between obesity and the 
infection caused by the bacterium Helicobacter pylori, which is causally related to gastric and duodenal cancer, and is recognized by IARC as a carcinogen (22).

Another evidence confirming the association of intestinal microflora with the process of cancer is research on Fusobacteria. Their increased amount was observed in the saliva of women with obesity. The studies showed an increased amount of these bacteria in patients with colorectal cancer $(22,23)$.

\section{Method of Estimating Risk of Cancer Incidence in People with Excessive Body Mass}

Despite the evidence showing the undoubted influence of obesity on the incidence of cancer, there is relatively little data on the impact of weight gain or loss on changes in the risk of cancer. Lack of data on weight loss can cause a small number of people able to achieve permanent loss of excess body weight. Identification of risk factors and estimation of the following parameters: body mass index (BMI), body fat percentage, then the odds ratio (OR) calculation in relation to the risk of developing selected types of cancer, adjusted for age, sex, race/ethnicity and place of residence should be a helpful tool. The risk assessment is carried out on the basis of results obtained in epidemiological analytical (cohort) studies and case-control and experimental studies.

Cohort studies consist of observation of two groups of people exposed and not exposed to the examined risk factor. The starting point is therefore the risk factor (Table below). The examination usually lasts for many years and consists of assessing the frequency of occurrence of the disease in question in people in both groups.

When estimating the increase in the odds ratio of selected malignant tumours as well as the range in which the quotient value increases significantly or decreases (24). The odds ratio should be determined for individual risk factors.

\begin{tabular}{|l|c|c|c|}
\hline Risk factor & Does occur & Does not occur & Altogether \\
\hline Test group & $a$ & $b$ & $a+b$ \\
\hline Control group & $c$ & $d$ & $c+d$ \\
\hline Altogether & $a+c$ & $b+d$ & $a+b+c+d$ \\
\hline
\end{tabular}

Calculating the chance of developing a selected malignant tumour when the risk factor occurred:

$$
\text { Chance }_{\text {yes }}=\frac{\frac{a}{a+c}}{1-\frac{a}{a+c}}
$$

and when it did not occur:

$$
\text { Chance }_{\text {no }}=\frac{\frac{b}{b+d}}{1-\frac{b}{b+d}}
$$

Calculating the odds ratio (OR) with $95 \%$ confidence interval:

$$
O R=\frac{a * d}{c * b}
$$

\section{Prevention of Cancer}

The best way to prevent cancer caused by overweight or obesity is to achieve and maintain a healthy body weight. So far, however, there is no accurate scientific confirmation of the extent to which weight reduction can protect against cancer, because only a few patients can effectively reduce and maintain normal BMI values for a longer period of time. The only evidence is based on the study of patients after bariatric surgery (25). People who have undergone surgical treatment of obesity have a lower risk of cancer compared to people who did not undergo such an operation. The reduction diet and lifestyle changes can reduce up to $10 \%$ of body weight, while $30 \%$ of excess body weight can be reduced as a result of bariatric procedures. The level of hormones that stimulate the development of cancer is reduced by a slight reduction in body weight. The relationship between the reduction of overweight and obesity and the reduction of the risk of developing postmenopausal breast cancer is increasingly known (26).

Weight reduction, especially before menopause, is a protective factor against future breast cancer. Endocrine and metabolic disorders related to overweight are reversible through effective weight reduction, which is recommended to lower estradiol and estrone, lipids, leptin, insulin, and inflammatory cytokines. In addition to the reduced risk of cancer, weight reduction can also protect against other chronic non-infectious diseases - type 2 diabetes or heart disease. Many people have a serious problem to maintain a healthy weight. Even if a reduction is achieved for some time, they usually return to their previous body weight. It is so-called yo-yo effect, which can also stimulate cancer development. In IACR studies, body weight fluctuations may increase the risk of kidney cancer $(1,7)$.

The Swedish obesity study showed that under the influence of effective treatment of obesity, a reduction in overall long-term mortality by $29 \%$ (hazard ratio, $\mathrm{HR}=0.71$ ) can be achieved. This was accompanied by a reduction in the incidence of cancer (27).

Obesity and poor nutrition are attributed to $30 \%$ of the effect on the occurrence of cancer, as well as smoking, in total, lifestyle in $60 \%$ determines the development of cancer. Obesity increases the risk of cancer 1-2 times, along with the increase in BMI also the risk of death due to cancer. In people with BMI $\leq 40 \mathrm{~kg} / \mathrm{m}^{2}$, the risk increases more than 3 times compared to people with normal BMI (26).

Due to the proven dependence between the development of cancer and obesity, anti-cancer prevention should be considered as avoiding and fighting obesity, especially since this problem is increasingly affecting children and young people (2).

Liver cancer accounts for about $7 \%$ of cases worldwide. Chen et al. showed that BMI $\geq 25 \mathrm{~kg} / \mathrm{m}^{2}$ is associated with the risk of primary liver cancer. The exact mechanism that promotes the development of this cancer is not fully understood, but it is certain that it is affected by non-alcoholic fatty liver (can lead to cirrhosis and then cancer of this organ), which is very common in obese people and is associated with insulin resistance (28).

In obese women, especially after menopause, the risk of developing breast cancer and its recurrence increases. Also, the risk of death from this disease is 2.5 times higher in such people than in lean women. As reported by Chan et al., An increase in the BMI index of $5 \mathrm{~kg} / \mathrm{m}^{2}$ (before the diagnosis of cancer) increases the mortality risk by as much as $17 \%$ (28). Larsson and Wolk investigated the relationship between obesity and the 
occurrence of Hodgkin's lymphoma. The authors suggest that with each increase in the BMI index by $5 \mathrm{~kg} / \mathrm{m}^{2}$, the risk of nonHodgkin's lymphoma increased by 7\%; mortality for this reason also increased by $14 \%$ (29).

In Europe, colorectal cancer takes first place among infections. The relationship between high BMI values and the risk of developing colorectal cancer is well documented. In diabetic patients, the risk of colon cancer increases in both men and women. In the study by Niebisz et al., among women with diabetes $18.5 \%$ suffered from colorectal cancer, while among diabetic men $-10.8 \%$ of them struggled with this cancer (30). As indicated by the American Institute for Cancer Research and the World Cancer Research report, obesity causes the development of many cancers. Of these, a definite percentage are cancers of the gastrointestinal tract (26).

Another important factor in the prevention of cancer is regular physical activity. The human body is adapted to physical activity. However, over the past dozen or so years, this activity has clearly decreased. IACR recommendations emphasize that moderate activity lasting a minimum of 30 minutes, repeated at least 5 times a week, is most beneficial $(1,2,8,26)$.

The results of research conducted in Great Britain show that passive lifestyle is responsible for $4 \%$ of cases of colon cancer, endometrium and breast cancer. Regular, moderate activity (30-60 $\mathrm{min}$ /day) reduces the risk of breast cancer, which is confirmed by many studies (26). In one meta-analysis 47 studies have found that exercise reduces the risk of breast cancer by $25-30 \%$ in women both before and after menopause (25).

There are many mechanisms explaining the relationship between physical activity and the risk of breast cancer. In addition to reducing body fat content and modifying estrogen levels, exercises also affect the regulation of ovulatory cycles, so that endogenous steroids affect the breasts of women to a lesser extent. The movement also leads to increased immune response, lowering insulin concentration and IGF-1 growth factor, and is responsible for regulating the activity of enzymes exhibiting inhibitory properties for free oxygen radicals. A Swedish study from 2008 conducted in a group of 40,000 men shows 34\% lower risk of death caused by cancer for people who walked or rode every day for a minimum of half an hour. The most scientific confirmation of the relationship between lack of exercise and cancer risk is noted in the case of colon cancer. Increased incidence of gastrointestinal cancer may result from excessive supply of calories, and low physical activity and sedentary lifestyle. Physical exercises can reduce the risk of getting sick for a quarter, because the amount of visceral fat decreases through movement. The movement regulates the digestive tract - improves intestinal peristalsis. Thanks to this, carcinogens contained in undigested food leftovers that could initiate cancerous changes leave the intestines faster. Exercise reduces inflammation of the intestine, which over time can contribute to the development of cancer. There are also a number of other mechanisms by which physical activity can protect against colorectal cancer - reduces insulin resistance and affects the metabolism of endogenous steroid hormone (25-27).

\section{CONCLUSION}

According to the current state of knowledge, the modification of factors significantly affecting the risk of disease can contribute to reducing the risk of cancer in obese patients. In view of the above, the importance of the problem of overweight and obesity in society and their impact on the occurrence of some types of cancer should be emphasized. Thus, the understanding of overweight and obesity as a serious health and social problem sets one of the priorities in the area of public health. The main intended scientific effect of such research will provide information on making further therapeutic decisions in the treatment of obese patients in the context of its compliance with current knowledge based on scientific literature. The main intended practical effect is to assess the risk of patients with excessive body weight. Individual risk measurement may result in selecting a group with an increased risk and taking appropriate actions, thus striving to reduce mortality. Better defined health needs of patients with obesity will have a better chance of responding adequately to case law, social support groups and, consequently, more effective help. In addition, learning about the intensity of individual cancer risk factors creates the opportunity to prepare more effective educational and preventive programmes, aimed at primary and secondary prevention of cancer and rational use of funds allocated to the above activities. Knowledge about activity of these factors in particular types of cancer can be very valuable information for planning targeted treatment, which is currently the subject of research in the world.

\section{Author's Contribution}

K.K.K. - study design; K.K.K., D.D. - data collection, data interpretation; K.K.K. - manuscript preparation, translation; K.K.K., D.D. - literature research; K.K.K. - final approval of the version to be published.

\section{Conflict of Interests}

None declared

\section{REFERENCES}

1. Bray F, Ferlay J, Soerjomataram I, Siegel RL, Torre LA, Jemal A. Global cancer statistics 2018: GLOBOCAN estimates of incidence and mortality worldwide for 36 cancers in 185 countries. CA Cancer J Clin. 2018;68(6):394-424.

2. World Health Organization. Obesity and overweight [Internet]. Geneva: WHO; 2020 [cited 2021 Jan 21]. Available from: https://www.who.int/ news-room/fact-sheets/detail/obesity-and-overweight.

3. NCD Risk Factor Collaboration (NCD-RisC). Worldwide trends in bodymass index, underweight, overweight, and obesity from 1975 to 2016: a pooled analysis of 2416 population-based measurement studies in 128.9 million children, adolescents, and adult. Lancet. 2017;390(10113):262742.

4. Calle EE, Rodriguez C, Walker-Thurmond K, Thun MJ. Overweight, obesity, and mortality from cancer in a prospectively studied cohort of U.S. adults. N Engl J Med. 2003;348(17):1625-38.

5. Renehan AG, Tyson M, Egger M, Heller RF, Zwahlen M. Body-mass index and incidence of cancer: a systematic review and meta-analysis of prospective observational studies. Lancet. 2008;371(9612):569-78.

6. Wolin KY, Carson K, Colditz GA. Obesity and cancer. Oncologist. 2010;15(6):556-65.

7. Ligibel JA, Alfano CM, Courneya KS, Demark-Wahnefried W, Burger RA, Chlebowski RT, et al. American Society of Clinical Oncology position statement on obesity and cancer. J Clin Oncol. 2014;32(31):3568-74.

8. Sung H, Siegel RL, Torre LA, Pearson-Stuttard J, Islami F, Fedewa SA, et al. Global patterns in excess body weight and the associated cancer burden. CA Cancer J Clin. 2019;69(2):88-112.

9. Fu J, Hofker M, Wijmenga C. Apple or pear: size and shape. Cell Metab. 2015;21(4):507-8.

10. Jung UJ, Choi MS. Obesity and its metabolic complications: the role of adipokines and the relationship between obesity, inflammation, insulin 
resistance, dyslipidemia and nonalcoholic fatty liver disease. Int J Mol Sci. 2014;15(4):6184-223.

11. O'Flanagan CH, Bowers LW, Hursting SD. A weighty problem: metabolic perturbations and the obesity-cancer link. Horm Mol Biol Clin Investig. 2015;23(2):47-57.

12. Divella R, De Luca R, Abbate I, Naglieri E, Daniele A. Obesity and cancer: the role of adipose tissue and adipocytokines induced chronic inflammation. J Cancer. 2016;7(15):2346-59.

13. Hursting SD, DiGiovanni J, Dannenberg AJ, Azrad M, Leroith D, Demark-Wahnefried W, et al. Obesity, energy balance, and cancer: new opportunities for prevention. Cancer Prev Res (Phila). 2012;5(11):126072 .

14. Kwiatkowska K, Pawłowska A, Suszczyk D, Bilska M, Tarkowski R, Kotarski J, et al. Does obesity increase the risk of ovarian cancer? A literature review. Curr Gynecol Oncol. 2017;15(4):277-83.

15. Fujita K, Hayashi T, Matsushita M, Uemura M, Nonomura N. Obesity, inflammation, and prostate cancer. J Clin Med. 2019;8(2):201. doi: $10.3390 / \mathrm{jcm} 8020201$.

16. Walczak K, Marciniak S, Rajtar G. Cancer chemoprevention - selected molecular mechanisms of action. Postepy Hig Med Dosw (Online). 2017;71(0):149-61.

17. Divella R, Mazzocca A, Daniele A, Sabba C, Paradiso A. Obesity, nonalcoholic fatty liver disease and adipocytokines network in promotion of cancer. Int J Biol Sci. 2019;15(3):610-16.

18. Espinoza JA, Bizama C, Garcia P, Ferreccio C, Javle M, Miquel JF, et al. The inflammatory inception of gallbladder cancer. Biochim Biophys Acta. 2016;1865(2):245-54

19. Lashinger LM, Ford NA, Hursting SD. Interacting inflammatory and growth factor signals underlie the obesity - cancer link. J Nutr. 2014;144(2):109-13.

20. Hopkins BD, Goncalves MD, Cantley LC. Obesity and cancer mechanisms: cancer metabolism. J Clin Oncol. 2016;34(35):4277-83.

21. Othman EM, Oli RG, Arias-Loza PA, Kreissl MC, Stopper H. Metformin protects kidney cells from insulin-mediated genotoxicity in vitro and in male zucker diabetic fatty rats. Endocrinology. 2016;157(2):548-59.
22. Rea D, Coppola G, Palma G, Barbieri A, Luciano A, Del Prete P, et al. Microbiota effects on cancer: from risks to therapies. Oncotarget. 2018;9(25):17915-27.

23. Castaner O, Goday A, Park YM, Lee SH, Magkos F, Shiow STE, et al. The gut microbiome profile in obesity: a systematic review. Int J Endocrinol. 2018;2018:4095789. doi: 10.1155/2018/4095789.

24. Pita B, Grodecka-Gazdecka S, Chmaj-Wierzchowska K, Kramer L, Opala $\mathrm{T}$. Analysis of the odds ratio for developing breast cancer of women. Ginekol Pol. 2011;82(10):755-60. (In Polish.)

25. Casagrande DS, Rosa DD, Umpierre D, Sarmento RA, Rodrigues CG, Schaan BD. Incidence of cancer following bariatric surgery: systematic review and meta-analysis. Obes Surg. 2014;24(9):1499-509.

26. World Cancer Research Fund International; American Institute for Cancer Research. Food, nutrition, physical activity, and the prevention of cancer: a global perspective. Washington: AICR; 2007.

27. Sjöström L. Review of the key results from the Swedish Obese Subjects (SOS) trial - a prospective controlled intervention study of bariatric surgery. J Intern Med. 2013;273(3):219-34.

28. Chen Y, Wang X, Wang J, Yan Z, Luo J. Excess body weight and the risk of primary liver cancer: an updated meta-analysis of prospective studies. Eur J Cancer. 2012;48(14):2137-45.

29. Larsson SC, Wolk A. Body mass index and risk of non-Hodgkin's and Hodgkin's lymphoma: a meta-analysis of prospective studies. Eur J Cancer. 2011;47(16):2422-30.

30. Niebisz AB, Kotarski P, Jasik M, Karnafel W. Diabetes and cancer. Diabetol Prak. 2008;9(2):89-93. (In Polish.)

Received August 3, 2019 Accepted in revised form February 21, 2021 